\title{
news news news news
}

\section{Depression worsens health of people}

with chronic illness

Individuals with depression are generally less healthy than those with a major chronic disease, according to data collected by the World Health Organization (WHO) in its World Health Survey on health and health-related outcomes and their determinants in adults aged 18 years and older and presented in the organisation's World Health Statistics 2007.

On a scale of $0-100$, reflecting the worst to best level of health, individuals without depression or other conditions had a mean health score of 90. Respondents with one of the chronic diseases - angina, arthritis, asthma or diabetes - had mean health scores of around 80 while those with depression but without chronic disease had mean health scores around 73. However, respondents with depression and another chronic condition had even lower mean health scores, around 63.

The report states that depression is an important global public health problem due to both its relatively high lifetime prevalence and the significant disability that it causes. In 2002, depression accounted for $4.5 \%$ of the worldwide total burden of disease as well as approximately $12 \%$ of total years lived with disability worldwide. Without treatment depression has the tendency to assume a chronic course, to recur, and to be associated with increasing disability over time.

The analysis was unable, however, to indicate whether people are more depressed because they have a coexisting chronic condition. Nevertheless the timely diagnosis and treatment of depressive disorders are essential irrespective of causality, and these can be treated in primary care or community settings using locally available and costeffective interventions.

Soutce: www.who.in

\section{Mental health and changing service delivery in South Africa}

Uncertainty about the delivery of services such as electricity and water in South Africa following the introduction of policy reforms around cost recovery and commercialisation is adding to the stress levels of lower income dwellers in the country's townships and rural areas, a new study from the Municipal Services Project has found.

The study entitled 'How depressing: Poverty, mental health and municipal services in South Africa' (Occasional Paper No. 12) found that in all of the households interviewed there was considerable financial hardship as a result of cost recovery strategies on basic municipal services, with $29 \%$ of household income being spent on water and electricity on average and arrears on water and electricity bills as high as R 18200 . Respondents expressed anxiety about this situation, noting that it added considerably to overall family stress. 


\section{news news news news}

However, the study was found to be too small and too preliminary to lead to firm conclusions about service delivery and mental disorders in general. Nevertheless the outcomes point to issues which bear further consideration, including:

- Health and safety problems: Household members have difficulties, due to lack of water, in ensuring appropriate use of medication, practising adequate hygiene and growing their own food. There are also concerns about being forced to use open fires, candles and paraffin stoves for cooking and warmth, leading to additional health and safety worries such as poisoning, fires, and respiratory infections.

- Time and energy: Considerable time and energy are spent searching for alternative sources of water and electricity and having to live with limited supplies of both.

- Social tensions: Respondents expressed concern with having to borrow money or water from neighbours and family members, leading to additional stresses in their lives and often to tensions within families and neighbourhoods.

- Social activities: Reducing service consumption has implications for people's social lives and household entertainment. Most of the households interviewed owned a television or radio, for example, but were reluctant to use them due to electricity costs. One family member reported being bored at home and therefore spending time with peers who encouraged him to use drugs.

- Relapse: The stress of not being able to afford adequate services, or having these services cut off or restricted, would appear to add considerable stress to the person with the mental disorder, possibly contributing to a worsening of the disorder and/or relapse.

- Impact on caregiving environment: Inadequate services would appear to increase levels of stress and burden for the caregiver with implications for their own mental health. This then impacts on the family member with the mental disorder as well as the household as a whole, as the caregiver's ability to care for the family may be compromised. Women appear to be the most affected by this as the primary caregivers.

Addressing these concerns will require consideration across a wide range of policy fronts, says the report. 'From funding for disability grants to the price of municipal services, the allocation of free water and electricity supplies, policies on prepaid meters and service restrictions, and dealing with the stigmatisation of mental disorder, improving the situation for low-income households with mental health concerns will not be an easy or simple task and will require substantial interdepartmental collaboration as well as interdisciplinary research and dialogue.'

Source: www.queensu.ca/msp 